\title{
Scolia
}

Revue de linguistique

30 | 2016

Des connecteurs argumentatifs aux opérateurs discursifs

\section{Les marqueurs en voir : de la fonction d'appel à la fonction épilinguistique}

Le cas des formes impératives vois / voyons / voyez

Jean-Claude Anscombre

\section{OpenEdition \\ Journals}

Édition électronique

URL : http://journals.openedition.org/scolia/456

DOI : $10.4000 /$ scolia.456

ISSN : 2677-4224

Éditeur

Presses universitaires de Strasbourg

Édition imprimée

Date de publication : 19 juin 2016

Pagination : 15-32

ISBN : 978-2-86820-944-3

ISSN : $1253-9708$

\section{Référence électronique}

Jean-Claude Anscombre, «Les marqueurs en voir : de la fonction d'appel à la fonction épilinguistique », Scolia [En ligne], 30 | 2016, mis en ligne le 09 avril 2019, consulté le 24 mai 2019. URL : http:// journals.openedition.org/scolia/456 ; DOI : 10.4000/scolia.456 


\title{
Les marqueurs en voir: de la fonction d'appel à la fonction épilinguistique Le cas des formes impératives vois/voyons/voyez
}

\author{
Jean-Claude Anscombre \\ LDI CNRS UMR 7187 \\ janscombre@ldi.univ-paris13.fr
}

\section{Introduction}

Dans la mesure où ils résultent dans une large part de processus de lexicalisation de valeurs en contexte, les marqueurs discursifs peuvent a priori avoir n'importe quel point de départ pour leur formation ${ }^{1}$. Parmi les verbes utilisés, outre dire, à la base de la création de plus de cent marqueurs en français contemporain (cf. Rouanne \& Anscombre, 2016), les verbes dits de perception ont été mis à contribution: entendre (à vous entendre), écouter (écoute voir), regarder (regarde-moi ça), mais aussi voir, qui a engendré plusieurs marqueurs dont voici/voilà (Delahaie, 2013), tu vois (Bolly, 2010), voyons (Sirdar-Iskandar, 1983a; Léard, 1990), mais aussi je vois, je vois ça d'ici, j'aimerais vous y voir, faut voir, c'est tout vu, ça reste à voir, etc.

La présente étude se limite à l'examen des marqueurs discursifs centrés sur les formes impératives de voir, c'est-à-dire vois/voyons/ voyez, et signalés dès Anscombre (1985). On se propose ici d'établir non seulement leur caractérisation linguistique, en particulier sémantique,

1 On trouvera par exemple une hypothèse de formation de marqueurs comme s'il vous plaît, je vous en prie, hélas, etc., par acquisition puis lexicalisation d'une valeur pragmatique dès Anscombre $(1979,1981)$. 
mais aussi épilinguistique, ces marqueurs n'étant pas intégrés dans le discours, mais plutôt insérés (cf. Delofeu, 2014). A ce titre, on étudiera leur statut de parenthétique(s), et on tentera de caractériser les types de co-textes et de contextes dans lesquels ils peuvent apparaître. Un dernier paragraphe examinera la diachronie de ces marqueurs, en vue de déterminer les règles qui permettent la création d'un marqueur discursif par passage d'un sens purement lexical à une fonction d'articulation discursive.

\section{Quelques données de départ}

\subsection{Quelles formes impératives?}

Les formes impératives simples de voir étant vois/voyons/voyez, les candidats au statut de marqueur sont par conséquent: vois, voyons, voyez, voyons (un peu+ça+voir), vois/voyez donc, voyons donc, ben voyons, voyez-moi ça, voyez-vous ça. Toute la question est donc de distinguer les emplois réguliers de voir à l'impératif des formes impératives de voir qui se sont spécialisées en tant que marqueurs autonomes.

a) Une première constatation est que deux des éléments de la liste - à savoir voyez-moi ça, voyez-vous ça - font problème en tant que formes impératives. Dans le premier cas, l'interprétation comme un impératif assorti d'un pronom d'intérêt (datif d'intérêt) semble ne pas faire problème. Il y a des cas analogues: regardez-moi ça, faites-moi ça, raconte-moi ça, prends-moi ça, etc. Notons cependant qu'à regardez-moi ça correspond regardemoi ça, alors qu'à voyez-moi ça ne correspond pas un vois-moi $c ̧ a$, mais un vois ça. Il s'agit peut-être d'un indice de la formation d'un nouveau marqueur ${ }^{2}$.

b) Pour ce qui est de voyez-vous ça, la solution du datif éthique ne semble pas possible. En face de regardez-moi ça, goûtez-moi ça, etc., on a en effet ${ }^{*}$ regardez-vous ça, ${ }^{\star}$ goûtez-vous ça, etc. La possibilité de faites-vous ça provient d'une lecture réfléchie et n'atteste donc pas la présence d'un datif éthique. Il s'agit plus

2 Notons qu'on ne peut invoquer ici le monosyllabisme de vois comme origine du phénomène, puisqu'on a sans problème fais-moi ça. 
vraisemblablement d'une ancienne forme interrogative à valeur de demande de confirmation, ou encore de l'inversion propre à certaines incises (dont celles en dire). Ce qui expliquerait à la fois l'inversion et la valeur actuelle de prise à parti. Notons que la langue moderne n'utilise guère l'inversion pour cette valeur, mais la forme interrogative par intonation: t'as $v u$ ça, vous avez vu ça $a^{3}$. Diachroniquement, les formes vois-tu/voyezvous en incise avec valeur de demande d'accord, apparaissent dès le $\mathrm{xvI}^{\mathrm{e}}$ siècle en alternance avec d'autres formes comme ne vois-tu/voyez-vous pas, vois-tu/voyez-vous pas/point, i.e. des interrogations de type rhétoriques. Seule la forme voyez-moi ça entre donc de plein droit dans la liste des formes impératives que nous nous proposons d'étudier".

c) Une seconde constatation concerne les formes simples vois et voyez, que ce soit en combinaison (avec un complément d'objet ou une subordonnée) ou encore en incise. Elles ont la valeur impérative attendue, et sont d'ailleurs substituables par d'autres formes impératives de valeur proche: regarde/regardez, vérifie/ vérifiez, jette un coup d'œil/jetez un coup d'œil, va voir/allez voir, etc. Il ne s'agit donc pas de marqueurs, si du moins on pose que la formation d'un marqueur discursif passe par la perte, la modification ou le déplacement de tout ou partie des valeurs sémantiques de départ et l'acquisition de valeurs pragmatiques à l'origine non intégrées.

La liste des candidats au statut de marqueur discursif se réduit donc à: voyons (du moins certains emplois), voyons (un peu $+c ̧ a+v o i r)$, voyons donc, ben voyons, voyons voyons voyez-moi ça. Nous ferons une étude par forme, ce qui signifie entre autres que: a) s'il faut toujours tenter de réduire au maximum la multiplication des entités théoriques pour une même forme, ce n'est pas toujours possible; b) d'une même forme peuvent être dérivées deux formes distinctes qui, même si elles sont visiblement parentes, ne peuvent pas toujours être ramenées à une

3 Avec le parallèle t'entends ça/t'as entendu ça/vous entendez ça/vous avez entendu ça face à un entendez-vous ça plutôt rare.

4 Il est donc d'autant plus remarquable de voir que voyez-moi ça et voyez-vous ça issues de formes impérative et interrogative respectivement aboutissent à des fonctions apparemment proches en tant que marqueurs. 
fonction de base unique. Nous sommes en présence d'un tel cas avec voyons donc et voyons voyons, comme nous le verrons plus loin.

\subsection{Quels marqueurs?}

Nous avons réussi à séparer les formes non impératives de celles qui sont impératives ou proviennent de formes impératives. Un dernier point consiste à séparer celles des formes restantes qui sont de simples impératifs de celles qui sont d'authentiques marqueurs discursifs. Ce qui suppose de spécifier quelles propriétés minimales nous paraissent être compatibles avec l'hypothèse d'un statut de marqueur discursif.

Certaines propriétés semblent en effet caractériser le statut de marqueur, et plus particulièrement la parenthétisation ou la mise en incise, et donc son émergence comme marqueur ainsi que les propriétés qui l'accompagnent fréquemment, du moins dans nos langues. En particulier, comme souvent noté: a) une valeur sémanticopragmatique faisant intervenir l'attitude du locuteur; b) une autonomie syntaxique plus ou moins marquée par rapport au reste du texte dans lequel il intervient; c) une autonomie prosodique par rapport au reste du texte $\mathrm{e}^{5}$ d) une perte du caractère référentiel de certains composants. Dans le cas (fréquent) d'un parenthétique, ce peut être le caractère non-référentiel d'un pronom ou la perte de ce pronom: le en de je vous en prie 'de rien' correspond au premier cas. L'absence de pronom de je vous prie 's'il vous plait', au second. En revanche, l'absence de pronom de je crois s'oppose à la présence d'un pronom-copie dans je le crois ${ }^{6}$; e) une autonomie sémantique plus ou moins marquée par rapport aux emplois non parenthétiques, par exemple une complétive en $q u e^{7}$; f) une grande mobilité de position, qui peut être initiale, médiane ou finale. La position initiale n'est cependant pas toujours possible: elle l'est pour $t u$ sais mais non pour $t u$ dis. Nous serons en revanche très prudent quant aux hypothèses de figement, dont on a usé et abusé,

5 Cf. Anscombre (2013a) pour les deux intonations - montante et descendante - de bien sûr dans les structures bien sûr, $p$. Sur la prosodie des incises, cf. Gachet-Avanzi (2008), Gachet (2009).

6 Cf. Anscombre (1981) sur ces problèmes de dislocation avec ou sans copie. Comme on (le) voit, ces problèmes ne sont pas nouveaux.

7 Cf. Anscombre (2013a) pour bien sûr vs bien sûr que de ce point de vue, et Delahaie (2014) pour heureusement vs heureusement que. 
et qu'on a parfois confondu avec la présence d'une matrice lexicale (Anscombre, 2011).

\subsection{Un échantillonnage}

Voici un bref aperçu du type de données que nous envisageons de traiter.

1) [...] écarquillés, puis, pour ne pas être de reste, tenta de se mettre à l'unisson en beuglant aussi fort qu'eux. Elle y parvint presque. - Voyez-moi ça, voyez-moi ça, fit l'un des mecs. On lui a fait peur, à la petite mignonne? (Benoziglio, Cabinet portrait, 1980)

2) [...] il continuerait d'appeler au calme d'une voix mesurée: Allons, messieurs! Voyons, madame!, tout en songeant à la bombe lacrymogène dans le tiroir sous la caisse dont il aurait bien aspergé tout le monde... (Jenni, L'Art français de la guerre, 2011)

3) [...] On ne polémiquera pas sur le désir de sécurité, bien entendu légitime... Le problème est ailleurs: dans la montée, de plus en plus perceptible, de la confusion et du conformisme intellectuels. Voyons ça, transversalement, de plus près. (Corpus Le Monde, 2007-2013)

4) [...] Voyons un peu quand même ce que les individus sont capables de faire pour mettre en avant leurs petites personnes. Limitons nous à deux... (Roubaud, La Dissolution, 2008).

5) - Mais si, ce que vous écrirez sera très bien - Je n'ai pas beaucoup de temps en ce moment... - On peut attendre... voyons voir... On calcule. Je promets de réfléchir. Je refuserai. (Queneau, Journaux, 1914-1965/1996) Tout le monde t'attend. - Tout va bien? lui demanda son hôtesse en lui désignant un fauteuil.

6) - Oui, merci. - Vous êtes sûr? - Fatigué... Ben voyons... Elle avait bon dos, la fatigue... (Gavalda, La Consolante, 2008)

7) Marc Chadourne me donnait rendez-vous dans un an. Voyons donc où jien étais, en avril 1939... Quatre-vingts pages de journal pour ce seul mois d'avril 1939. (Mauriac, Signes, rencontres et rendez-vous, 1983)

8) Pleureurs et pleureuses, vous avez raison, tout finit mal, surtout les victoires. Voyons, voyons, restons calmes: qu'est-ce qu'on risque, après tout? Violation de domicile, déprédations 
volontaires, entrave à la justice. (Pennac, Monsieur Malaussène, 1995)

Nous nous proposons des qualifier les différentes fonctions que remplissent ces constructions, et d'examiner au passage dans quelle mesure elles peuvent être considérées comme des marqueurs ou à tout le moins en train d'évoluer vers des marqueurs.

\section{Le cadre théorique}

Nous ne retiendrons a priori ni la thèse d'une permanence d'une valeur centrale pour les marqueurs (thèse en général mise à mal par les données diachroniques) ni celle d'une désémantisation chère à d'autres. Non plus que la notion de verbe de perception, qui confond le mot de la langue, le mot de la métalangue relatif à une classe linguistique de verbes, et enfin le mot utilisé en sciences pour désigner une classe de phénomènes psycho-physiologiques ${ }^{8}$. Tracé à grands traits, notre approche se situe dans le cadre d'une pragmatique intégrée (Anscombre \& Ducrot, 1983). La valeur sémantique fondamentale d'un terme ou d'une combinaison de termes peut comprendre de façon constitutive des renvois à des potentialités autres que vériconditionnelles: des valeurs d'action, mais aussi des attitudes, concernant une situation ou même une énonciation (fonction épilinguistique). Cette valeur sémantique sera caractérisée dans un cadre polyphonique minimal, à savoir le suivant: tout discours - et en particulier tout énoncé - est caractérisé par un ensemble de points de vue ou $p d v$ (Anscombre, 2013b) dont la source peut être multiple. Un discours / énoncé fait sens en distribuant les différents acteurs discursifs (locuteur, interlocuteur, destinataire, etc. $)^{9}$ par rapport à ces $p d v$.

Dernier point: nous mettons au centre de notre étude la fonction d'appel propre en particulier à l'impératif. Bühler (1950: 70 sq.) voit ainsi la signification comme composée de trois fonctions fondamentales et indépendantes: de représentation ('description' du

8 Nous différons donc sur ce point de l'approche de Bolly (2010) dans son étude de tu vois.

9 Rappelons que ces acteurs ne sont pas des personnages réels, mais des entités que le discours lui-même caractérise comme étant l'auteur, le récepteur, etc., de ce discours. Elles sont donc construites par le discours. 
monde), d'expression (concernant la subjectivité du locuteur), et enfin d'appel (centrée sur l'allocutaire), l'ordre étant l'illustration exacte de cette dernière. Si la fonction de représentation semble peu pertinente ici, les deux autres sont en revanche au cœur du débat: la fonction d'appel puisque propre à un impératif originel, mais aussi la fonction d'expression, la modalisation opérée par un marqueur relevant de l'attitude et donc de la subjectivité du locuteur. Sirdar-Iskandar (1983b: 31) se fonde sur Bühler pour classer les interjections en deux classes: "celles qui expriment un état du locuteur [...] et celles qui servent à demander quelque chose à l'allocutaire»

Toute la question sera donc pour nous de spécifier, dans la description de chacun d'entre eux, ce qu'il est advenu de la fonction d'appel de base de l'impératif. Faute de place, nous nous contenterons des principales caractéristiques illustrées d'exemples.

\section{Analyse des marqueurs}

\subsection{Voyez-moi ça}

Les principales caractéristiques formelles en sont les suivantes:

a) Il s'agit - une fois n'est pas coutume - d'une forme presque totalement figée. En effet, vois-moi ça n'existe pas, non plus que voyez-nous ça. On ne peut supprimer le datif d'intérêt moi sans changer radicalement le sens et revenir alors à une valeur impérative habituelle. Enfin, si le ça n'est pas totalement obligatoire - il y a des cas comme Voyez-moi ce petit prétentieux! - ils semblent se raréfier. D’une part, Voyez-moi ça gagne du terrain, et d'autre part, surtout à l'oral, c'est la tournure T'as vu X - dont T'as vu ça est issue - qui s'impose.

b) Le schéma intonatoire est de type ( $\searrow$ ) (le type exclamatif de Quelle belle journée!), alors que T'as vu ça a de préférence le schéma montant $(\nearrow)$ (le type exclamatif de C'est un scandale!), bien que $(\searrow)$ ne soit pas impossible.

c) Il est proche par le sens de Voyez-vous ça!, qui lui est substituable, et qui est également pourvu du schéma $(\searrow$ ), à l'exclusion du schéma montant $(\lambda)$.

d) L'énoncé Voyez-moi ça n'est jamais un énoncé d'ouverture: il est toujours proféré en réaction à une énonciation ou à une 
situation. On le trouve d'ailleurs toujours devant une situation donnée, ou après une énonciation effective. Ainsi :

9) Au lieu de me transformer en sensualiste classique, donc manipulable, je réfléchis, je pourrais même dire que je pense. Oh, il pense! Voyez-moi ça! (Sollers, Le Secret, 1993).

e) Enfin, Voyez-moi ça peut apparaître sans être suivi d'un énoncé, commentaire ou autre. Mais plus ordinairement, il est suivi d'une évaluation de la situation ou de l'événement rapporté dans le discours antérieur:

10) - Un accident de rugby, sans doute!

Non, dit l'infirmier. Il a voulu se suicider en se jetant par la fenêtre de son dortoir, à Marbot...

- Voyez-moi ça! Le païen! Le mécréant! (Gibeau, Allons z'enfants, 1952)

Ça garde donc sa fonction déictique : il renvoie à la situation désignée ou décrite. Il nous reste maintenant à expliciter le fonctionnement linguistique de Voyez-moi ça et à caractériser les situations dans lesquelles il est utilisable. De façon générale, Voyez-moi ça apparaît dans la structure suivante: le locuteur $L$ de Voyez-moi ça se trouve face à une situation $S$ qui peut être soit une situation pure (i.e. non verbalisée), soit une situation constituée par divers énoncés dont il est ou non le locuteur. Il réagit à cette situation par Voyez-moi ça suivi d'énoncés qui peuvent être: a) rien du tout; b) un rappel d'un ou plusieurs énoncés antérieurs; c) une évaluation de $S$; d) un mélange de b) et de c). Nous caractériserons le fonctionnement de Voyez-moi ça à l'aide des traits sémantico-pragmatiques que $S$ doit satisfaire pour en autoriser l'emploi. Nous nous inspirons pour ce faire de l'étude de $\grave{a}$ coup sûr/bien sûr (Anscombre, 2001, 2013a, 2013b).

(i) Le trait $[\mathrm{d}]=L$ découvre $S$

Rappelons la définition de ce trait. Le locuteur $L$ d'un énoncé $\mathrm{E}$ dit découvrir une situation $S$ s'il présente une propriété, un objet, un événement présent dans $S$ comme: a) Faisant l'objet d'une expérience directe pour lui; b) Dans le contexte de l'énonciation de E, $L$ se présente comme ayant sa première expérience de cette caractéristique de $S$, et il ne s'agit pas par exemple d'une déduction tirée d'indices. Nous allons exposer quelques arguments montrant que voyez-moi ça fait intervenir 
$[+\mathrm{d}]$. Voyez-moi ça est possible si $L$ découvre $S$, impossible si $\mathrm{S}$ était antérieurement connue de $L$, comme illustré par:

11) A (à sa fille): - Tu restes à la maison ce soir? B : - Non, je vais au cinéma avec des copines.

A: - Voyez-moi ça! Et avec quelle permission, s'il te plaît?

12) Max m'envoie toujours un cadeau pour mon anniversaire. (À coup sûr + ??voyez-moi ça), il a le cœur sur la main.

Dans (12), à coup sûr est possible en tant que S satisfait [-d] (Anscombre, 2001), alors que voyez-moi ça est pour le moins bizarre, sauf à lire (12) comme un dialogue.

(ii) Le trait [i] = S n'était pas prévu par $L$

Nous le montrerons sur l'exemple (9) ci-dessus, plus précisément à partir du segment il pense! Voyez-moi ça!. L'auteur feint de découvrir (d'où l'ironie), au travers de je pourrais même dire que je pense que son double s'estime 'pensant', ce dont il se moque. Cet inattendu est marqué dans (9) par un $O h$ de surprise, mais peut l'être par d'autres indications:

13) $(\mathrm{Oh}+$ tiens + tiens donc + ça alors + je n'en reviens pas $+\ldots)$, il pense! Voyez-moi ça!

Y compris des indications d'un registre plus familier: sans blague, sérieux, t’as vu ça, t'entends ça, vise le mec!, etc.

(iii) Le trait [a] $=L$ en appelle à l'entourage comme garant de [i]

Ce trait peut se gloser comme suit: $L$ découvre $S$ auquel il ne s'attendait pas, et se justifie de son étonnement en demandant à l'entourage de constater, comme lui, la réalité de S. D'une part, l'entourage dont il s'agit est fictif et anonyme. On pourrait à ce propos invoquer la notion de $\mathrm{ON}$-destinataire qui ferait pendant à celle de ON-locuteur. D'autre part, on remarque que le marqueur voyez-moi ça, dans les termes de Bühler (1950), cumule les deux fonctions d'appel et d'expression. D'expression, car centré sur la subjectivité de $L$ - marquée par moi dont il exprime la surprise. D'appel à constat des lieux d'autre part - ce qui reste de l'impératif initial. On peut le voir en notant que les meilleurs substituts salvo sensu sont des interrogations rhétoriques: vous avez vu ça, vous avez entendu ça, non mais vous entendez ça, etc., y compris voyez-vous ça. Mais voyez-moi ça est le seul dont la fonction expressive soit indiquée par un pronom de $1^{\text {re }}$ personne. 
Enfin, il ne s'agit pas d'un parenthétique, mais d'une incise, et elle marque une attitude du locuteur par rapport à une situation que par ailleurs elle commente. Comme les adverbes d'énonciation et autres (Anscombre, 1990; Anscombre et alii, 2009), elle ne peut en effet être ni niée, ni interrogée, ni clivée, mais peut figurer en position frontale. Cependant, si elle peut figurer en position frontale ou médiane dans le texte définissant $S$, c'est en position finale qu'elle apparaît le plus souvent.

\subsection{Voyons: marqueurs et variantes}

Comme nous l'avons annoncé supra, voyons semble avoir plusieurs fonctions, et entre dans différentes combinaisons usuelles: ce sont voyons seul (du moins certains emplois), voyons (un peu + ça + voir), voyons donc, ben voyons, voyons voyons. Il s'agit alors de déterminer quand il y a marqueur (y compris en voie de formation) ou non, et si les différentes combinaisons sont ou non des variantes d'un même marqueur, avec le problème annexe de la possibilité ou non d'une valeur sous-jacente unique, dans le cadre théorique choisi, bien entendu.

\subsubsection{Le cas de voyons seul}

La forme voyons pose d'entrée les problèmes que nous venons d'évoquer. On peut distinguer quatre emplois principaux de voyons. C'est sur ce point que nous nous concentrerons, l'examen détaillé du fonctionnement de voyons ayant été fait dans (Sirdar-Iskandar, 1983a), même si nous ne partageons pas totalement ses vues. Ces quatre emplois sont illustrés comme suit:

$\left(\mathrm{E}_{1}\right) \quad$ Max, viens me donner un coup de main. Voyons si on peut sortir la voiture du fossé.

$\left(\mathrm{E}_{2}\right) \quad$ Voyons, qu'est-ce que je vais faire à manger ce soir?

$\left(\mathrm{E}_{3}\right) \quad \mathrm{A}:-\mathrm{Tu}$ viendras à la soirée demain?

B: - Bien sûr, voyons.

$\left(\mathrm{E}_{4}\right)$ Tiens-toi bien, voyons!

Le premier peut être qualifié d'emploi banal de voir. En effet, voyons y est accompagné d'une complétive, et ce pourrait être aussi bien 
un complément nominal: voyons ce qu'on peut faire de cette voiture. Par ailleurs, le singulier vois est possible. Enfin, ce pluriel englobe les deux participants (locuteur et destinataire) au discours, et voyons y a donc fonction d'appel - on peut lui ajouter sans problème toi et moi. Les trois autres exemples sont de ce point de vue fondamentalement différents. Vois y est impossible, quel que soit le nombre des êtres discursifs concernés, et le rétablissement d'une complétive ou d'un complément d'objet fait problème. Il s'agit donc d'une incise - et non d'un usage parenthétique. Les substituts possibles de voyons donnent quelques indications: examinons dans $\left(\mathrm{E}_{1}\right)$, réfléchissons dans $\left(\mathrm{E}_{2}\right)$, écoute dans $\left(\mathrm{E}_{4}\right)$. Le problème et plus délicat pour $\left(\mathrm{E}_{3}\right)$, où on pense à des choses comme la question ne se pose pas, la réponse est évidente. Le voyons de $\left(\mathrm{E}_{3}\right)$ et $\left(\mathrm{E}_{4}\right)$ comprendrait donc une fonction d'appel, la question restant posée pour $\left(\mathrm{E}_{2}\right)$. Par commodité, nous parlerons de voyons ${ }_{1}$ pour les cas tels que $\left(\mathrm{E}_{2}\right)$, et de voyons ${ }_{2}$ pour les cas tels que $\left(\mathrm{E}_{3}\right)$ et $\left(\mathrm{E}_{4}\right)$.

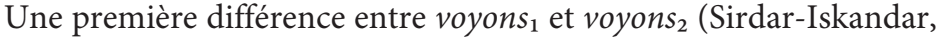
1983a) est que l'adjonction de un peu est banale dans $\left(\mathrm{E}_{2}\right)$ et impossible dans $\left(\mathrm{E}_{3}\right)$ et $\left(\mathrm{E}_{4}\right)$ :

14) Voyons un peu, qu'est-ce que je vais faire à manger ce soir?

15) A: - Tu viendras à la soirée demain?

$\mathrm{B}$ : - Bien sûr, ${ }^{*}$ voyons un peu.

16) Tiens-toi bien, *voyons un peu!

On a le même résultat avec voyons voir. Quelques remarques complémentaires:

a) En position postposée, ça/voir est possible comme complément de voyons $_{1}$, mais non de voyons ${ }_{2}$ :

17) Qu'est-ce que je vais faire à manger ce soir, voyons (ça + voir).

18) A: - Tu viendras à la soirée demain?

B: - Bien sûr, ${ }^{*}$ voyons $(c ̧ a+$ voir $)$.

19) Tiens-toi bien, ${ }^{*}$ voyons $(c ̧ a+$ voir $)$ !

b) Le français possède un usage de donc qui signale que l'énoncé où il apparaît est la conséquence de la situation ou d'une énonciation antérieures. D'où des exemples comme Dis donc, qu'est-ce que tu fais là? Ce donc est en fait possible tant avec voyons $_{1}$ qu'avec voyons $s_{2}$ : 
20) Voyons donc, qu'est-ce que je vais faire à manger ce soir?

21) A : - Tu viendras à la soirée demain?

B: - Bien sûr, voyons donc.

22) Tiens-toi bien, voyons donc!

On en déduit que tant l'un que l'autre sont compatibles avec une réaction à une situation ou une énonciation antérieures.

c) On remarque que $\left(\mathrm{E}_{1}\right)$ est réductible à une forme de type $\left(\mathrm{E}_{2}\right)$, mais non l'inverse:

23) Max, viens me donner un coup de main. Voyons, est-ce qu'on peut sortir la voiture du fossé?

24) Voyons si je vais faire à manger ce soir $\left(\neq E_{2}\right)$.

Une hypothèse possible est que voyons v $_{1}$ 'est formé à partir de l'impératif voyons par parenthétisation dans des exemples comme $\left(\mathrm{E}_{1}\right)$, puis a acquis une autonomie syntaxique qui l'a fait passer au statut d'incise.

d) La différence entre voyons v $_{1}$ et voyons 2 d'une part et le voyons de $\left(E_{1}\right)$ d'autre part peut être mise en évidence par le critère de coordination par et de deux actes de même nature (Anscombre, 1981; Sirdar-Iskandar, 1983a). Si on tente de coordonner dans chaque cas voyons avec un autre impératif:

25) Max, viens me donner un coup de main. Descendons et voyons si on peut sortir la voiture du fossé.

26) ??Regardons le livre de cuisine et voyons, qu'est-ce que je vais faire à manger ce soir?

27) A : - Tu viendras à la soirée demain?

B: - Bien sûr, ${ }^{*}$ voyons et crois-moi.

28) Tiens-toi bien, ${ }^{*}$ voyons et fais ce que je te dis! ${ }^{10}$.

e) Dernier point enfin: le voyons de $\left(\mathrm{E}_{1}\right)$ a très régulièrement une intonation montante, alors que voyons $s_{1}$ et voyons vavorisent $_{2}$ l'intonation descendante. Nous n'avons pu trouver aucun exemple de type $\left(\mathrm{E}_{4}\right)$ (le voyons dit de reproche) avec intonation montante.

Examinons maintenant diverses caractéristiques.

10 À ne pas confondre avec: Tiens-toi bien voyons, et fais ce que je te dis. 
(i) La fonction d'appel

Sa présence est claire dans les cas comme $\left(\mathrm{E}_{4}\right)$, où voyons est destiné à demander un faire au destinataire. Dans ce rôle, voyons peut être accompagné d'un impératif, d'un terme d'appel (Messieurs, voyons!) ou concerner une situation incluant le destinataire. Par exemple, on reprend un enfant qui mange avec ses doigts en lui disant Voyons! Le voyons de $\left(\mathrm{E}_{3}\right)$ (voyons dit d'interrogation) est essentiellement dialogique: il figure dans une réplique, où il est fréquemment accompagné d'expressions comme naturellement, bien sûr, bien entendu, indiquant que l'événement considéré doit être vu comme inéluctable. Voyons spécifie alors qu'il doit être vu ainsi par l'autre: la réplique de $\mathrm{B}$ pourrait de ce point de vue être accompagnée de commentaires comme qu'est-ce que tu crois?, tu devrais le savoir, la réplique entière pouvant d'ailleurs être remplacée par $\grave{A}$ ton avis? Autant de tournures qui font explicitement de l'auditeur un destinataire. La fonction d'appel figure donc bien au rang des caractéristiques de voyons $_{2}$. Qu'en est-il pour voyons v $_{1}$ ? Notons tout d'abord que cet emploi est compatible avec un impératif destiné à l'interlocuteur ou englobant le locuteur et l'interlocuteur: Voyons, donne-moi une idée pour le repas de ce soir et Voyons, cherchons ensemble une idée pour le repas de ce soir, par exemple. L'idée que nous avancerons est que, un peu à l'instar de voyez-moi ça, le locuteur de voyons 1 prend à parti l'entourage - réel ou fictif - en le faisant complice de sa situation. Là encore, une fonction de $O N$-destinataire conviendrait parfaitement. L'opposition entre voyons et écoute nous fournira un argument en faveur de notre thèse. Selon Sirdar-Iskandar (1983a), voyons! en général renvoie à quelque chose d'évident, d'admis par l'interlocuteur. En revanche, écoute! introduit un fait inconnu de l'interlocuteur et que lui apprend le locuteur. Imaginons que le locuteur ait à avouer à son interlocuteur qu'il est l'auteur d'une action préjudiciable à son égard. Il pourrait alors dire Écoute, j'ai un aveu pénible à te faire, mais certainement pas Voyons, j'ai un aveu pénible à te faire. Dans de telles circonstances en effet, l'interlocuteur ne peut être complice de la situation embarrassante où se trouve le locuteur. On aurait en revanche sans problème Voyons, j'avais autre chose à te dire, mais j'ai oublié quoi. Ce qui intéresse ici, c'est que Écoute! est à la deuxième personne du singulier alors que Voyons! est à la première personne du pluriel: cette différence est selon nous significative. 
(ii) Première personne ou pas?

Selon Sirdar-Iskandar (1983a: 113), la forme impérative en -ons est fréquemment utilisée pour une action qui est «...celle de l'autre...», et l'interjection voyons! manifeste constamment cette tendance à «... annuler la première personne...». On me permettra de soutenir la thèse inverse: il convient en effet de distinguer 'utiliser une première personne pour effectuer un acte effectuable à l'aide d'une deuxième personne', et 'annuler la première personne'. Si je vois arriver des invités, je pourrai leur crier par la fenêtre Entrez!, certainement pas Entrons! Quant au chef de gare qui dit aux voyageurs Montons! au lieu de Montez!, ce n'est pas (uniquement) en tant que rappel d'ordre, comme l'analyse Sirdar-Iskandar. La première personne atténue ce que l'ordre à la deuxième personne peut avoir de brutal, et c'est bien parce qu'elle inclut le locuteur que cette atténuation se produit. La police arrêtant un contrevenant lui dira, rappel d'ordre ou pas, Allez, sortez de la voiture et non pas Allons, sortons de la voiture. Dans le cas de voyons, nous allons le voir, ce maintien de la première personne dans la sémantique du marqueur provient de la mise en jeu d'une communauté linguistique - un $O N$-destinataire - dont fait partie le locuteur lui-même, du moins quant à certains principes.

(iii) Une fonction unique

Nous voudrions maintenant présenter un 'modèle' unique à la fois pour voyons $s_{1}$ et pour voyons $s_{2}$. Les exemples de type $\left(\mathrm{E}_{2}\right),\left(\mathrm{E}_{3}\right)$ et $\left(E_{4}\right)$ reposent donc pour nous fondamentalement sur le même fonctionnement de voyons, à savoir le suivant:

a) Voyons marque une attitude du locuteur $L$ par rapport à un certain état du monde $S$ : soit une situation qu'il constate, soit un fait faisant l'objet d'une énonciation.

b) Pour $L, S$ n'est pas l'état du monde tel qu'il le souhaite ou qu'il trouve normal.

c) Cette normalité est issue d'un principe soit général soit que $L$ présente comme tel (un $\mathrm{ON}$-locuteur).

d) $L$ prend à témoin une communauté linguistique dont l'interlocuteur fait partie (un $O N$-destinataire) de cet état du monde non conforme à la normalité.

e) $L$ demande à l'interlocuteur de rétablir la normalité quant à l'état du monde. 
Le point a) a déjà été évoqué dans sa quasi-totalité dans ce qui précède. Pour ce qui est de marquer une attitude, on note que de façon très générale, voyons ne supporte ni l'extraction, ni la négation, ni l'interrogation, caractéristiques communes aux marqueurs d'attitude (en particulier les adverbes d'énonciation), comme expliqué dans Anscombre (2001). Le point b) est clair sur des exemples comme $\left(E_{4}\right)$, où l'injonction de $L$ envers le destinataire d'avoir à réaliser $p$ montre que la situation $S$ comporte non- $p$. Dans les exemples de type $\left(E_{2}\right)$, du fait de se poser à lui-même la question, $L$ déclare ignorer quel $\mathrm{x}$ lui permettra de dire 'je vais faire à manger $\mathrm{x}$ ce soir'. Et en le commentant par voyons, il s'incite à déterminer un tel $\mathrm{x}$. Le cas de $\left(\mathrm{E}_{3}\right)$ est plus complexe. La réponse Bien sûr, voyons, à la question posée est-ce que $p$ ? signale (à l'aide de Bien sûr) que $p$ est la réponse évidente, et que l'état du monde que reflète la question - elle manifeste un doute quant à $p$ n'en est pas la vision correcte, et qu'il convient donc de 'rectifier le tir'. La question n'avait pas lieu d'être. C'est le rôle de voyons, et notons que dans cet emploi, il est substituable par qu'est-ce que tu crois? Le point e) découle de ce qui vient d'être dit, et le point d) renvoie à la fonction de 'prise à témoin' que nous avons évoquée plus haut. Il reste le point c): $L$ demande un retour à la normalité au nom d'un principe général - ou présenté comme tel par $L$, le locuteur de voyons. Le cas de $\left(\mathrm{E}_{4}\right)$ est de ce point de vue parfaitement clair: Tiens-toi bien, voyons, renvoie à un principe général du type de 'Il faut respecter les règles de la bienséance'. Mais ce principe n'est pas arbitraire: il s'agit du reflet en langue - en fait d'un stéréotype - valide à un moment donné dans une société donnée, et qui renvoie à un $O N$-locuteur. $L$ appuie son injonction par un rappel de la norme, et d'une norme qui inclut le destinataire. La première personne joue un rôle dans l'affaire: on pourrait avoir allons en lieu et place de voyons, mais non pas allez. On note que sauf à faire du nonsense, on n'aurait pas des choses comme: Tiens-toi mal, voyons, ou Sois malhonnête avec toi-même, voyons, etc. Voyons peut d'ailleurs s'appuyer dans son fonctionnement sur une Loi de discours. Dans le cas de $\left(E_{3}\right)$, il s'agirait de la loi selon laquelle on ne pose pas une question dont on connaît la réponse, en particulier lorsqu'elle est évidente. En disant voyons dans ce cas particulier, $L$ s'étonne - sur la base de cette loi - de ce que le destinataire pose une question dont il connaît la réponse aussi bien que $L$. Dans le cas de $\left(\mathrm{E}_{2}\right)$ enfin, voyons renvoie à un principe comme 'Préparer les repas suppose de choisir les $\mathrm{x}$ qu'on 
va manger'. Il n'est donc pas nécessaire de distinguer pour l'instant du moins, différents types de voyons, comme cela est habituellement fait. Il est également inutile de postuler un voyons de reproche comme le fait Sirdar-Iskandar (1983a). La valeur de reproche ne vient pas de voyons, mais de la nature déontique du principe général sous-jacent, ainsi dans $\left(\mathrm{E}_{4}\right)$. Un énoncé comme Couvre-toi, voyons dans un contexte où il fait très froid, n'est pas un reproche, mais un conseil, bien qu'étant de la même forme que $\left(\mathrm{E}_{4}\right)$. Nous évitons ainsi de multiplier inutilement les entités.

\subsubsection{Voyons voyons et Ben voyons}

Les cas de voyons (un peu $+c ̧ a+$ voir) et de voyons donc ayant été examinés plus haut, seuls restent les deux dernières combinaisons voyons voyons et ben voyons. Pour ce qui est de voyons voyons, on le rencontre sous les deux formes voyons $(\nearrow)$ voyons $(\searrow$ ) et voyons $(\rightarrow)$ voyons $(\searrow)$. Il est possible que la seconde possibilité indique un marqueur en formation, mais il convient d'être prudent. On a les parallèles de allons allons / allez allez, et il peut ne s'agir que du redoublement commun dans le domaine interjectif: tiens tiens, bon bon, écoute écoute, etc. Le cas de ben voyons semble plus clair. Sa fonction est indubitablement celle d'une réplique ironique: d'apparition récente (début $\mathrm{xx}^{\mathrm{e}}$ siècle), il se substitue à un eh bien, voyons rare avec cette fonction que n'a pas facilement non plus voyons seul. Il semble y avoir un paradigme: ben tiens, ben dis donc, ben vas-y, ce qui semblerait indiquer un marqueur à tout le moins en formation. Une étude détaillée reste à faire.

\section{Bibliographie}

ANSCOMBRE J.-C. (1979), Délocutivité benvenistienne, délocutivité généralisée, et performativité, Langue française 42, 61-84.

ANSCOMBRE J.-C. (1981), Marqueurs et hypermarqueurs de dérivation illocutoire: notions et problèmes, Cahiers de linguistique française 3, 75-123.

ANSCOMBRE J.-C. (1985), Onomatopées, délocutivité et autres blablas, Revue Romane 20: 2, 169-207.

ANSCOMBRE J.-C. (1990), Thème, espaces discursifs et représentations événementielles, in Anscombre J.-C. \& Zaccaria Z. (éds), Fonctionnalisme et pragmatique, Milan, Unicopli, 43-150. 
ANSCOMBRE J.-C. (2001), À coup sûr/Bien sûr: des différentes manières d'être sûr de quelque chose, in Dupuy-Engelhardt H., Palma S., Tyvaert J.E. (éds), Actes des Journées Scientifiques $2000, \mathrm{n}^{\circ} 16$, Les phrases dans les textes. Les sons et les mots pour le dire. Les connecteurs du discours. L'opposition verbo-nominale en acte, Reims, Presses Universitaires de Reims, 135-160.

ANSCOMBRE J.-C. (2009) \& al., Apparences, indices et attitude énonciative, Langue française 161, 39-58.

ANSCOMBRE J.-C. (2011), Figement, idiomaticité et matrices lexicales, in Anscombre J.-C. \& Mejri S. (éds), Le figement linguistique: la parole entravée, Paris, Champion, 17-40.

ANSCOMBRE J.-C. (2013a), À coup sûr et bien sûr et les fondements de la certitude, Revue de sémantique et pragmatique 33-34, 67-98.

ANSCOMBRE, J.-C. (2013b), Introduction: polyphonie et représentation sémantique des marqueurs de discours. Quelques problèmes, Revue de sémantique et pragmatique 33-34, 7-32.

ANSCOMBRE J.-C. \& DUCROT O. (1983), L'argumentation dans la langue, Bruxelles, Mardaga.

BOLLY C. (2010), Pragmaticalisation du marqueur discursif tu vois. De la perception à l'évidence et de l'évidence au discours, $C M L F$ 2010. http://www. Linguistiquefrançaise, 03/10/2014.

BÜHLER K. (1950), Teoría del lenguaje, Revista de Occidente, Madrid, Selecta.

DELAHAIE J. (2013), Vers une analyse sémantique (presque) unitaire des multiples emplois de voilà à leécrit et à l'oral, Revue de sémantique et pragmatique 33-34, 99-120.

DELAHAIE J. (2014), Les constructions en $a d v$. que $p$ : étude diachronique d sune tournure adverbiale particulière à partir du cas de heureusement que $p$, in Anscombre J.-C. OppermannMarsaux E. \& Rodríguez Somolinos A. (éds), Médiativité, polyphonie et modalité en français, Paris, Presses de la Sorbonne Nouvelle, 223-241.

DELOFEU J. (2014), La problématique de la liaison entre prédications à la lumière de la distinction entre construction et énoncé: intégration versus insertion, Langue française 2, 182, 59-73.

GACHET F. (2009), Les verbes parenthétiques: un statut syntaxique atypique?, $\operatorname{Lin} x$ 61, 13-29.

GACHET F. \& AVANZI M. (2008), La prosodie des parenthèses en français spontané, Verbum XXX: 1, 53-84.

LEARD J.-M. (1990), La sémantique de voyons: conséquences syntaxiques et pragmatiques, in Fall K. \& Souchard M. (éds), 
Discours, Représentation, Argumentation des savoirs et des formations, PUQ.

ROUANNE L. \& ANSCOMBRE J.-C. (éds) (2016), Histoires de dire. Petit glossaire des marqueurs formés sur le verbe dire, à paraître, Peter Lang.

SIRDAR-ISKANDAR C. (1983a), Voyons!, Cahiers de linguistique française de Genève 5, 111-130.

SIRDAR-ISKANDAR C. (1983b), Allons!, Semantikos 7: 1, 28-44. 